\title{
EMBEDDING OF CALABI-YAU DEFORMATIONS INTO TORIC VARIETIES
}

\author{
ANVAR R. MAVLYUTOV
}

\section{Contents}

1. Introduction.

2. Toric varieties, Calabi-Yau hypersurfaces and deformations. 3

3. Calabi-Yau deformations arising from weighted projective spaces. 4

4. Embedding of Calabi-Yau deformations using one edge.

References

\section{InTRODUCtion.}

In [M5], we have constructed non-polynomial deformations of Calabi-Yau hypersurfaces in toric varieties. Here, we will construct an embedding of such deformations into toric varieties. More precisely, we show that deformations of semiample (minimal) Calabi-Yau hypersurfaces in complete simplicial toric varieties can be realized as Calabi-Yau complete intersections in higher dimensional simplicial toric varieties. This result can be extended to all nef Calabi-Yau complete intersections as well.

There are several questions and conjectures which arise as a result of this construction. The deformations of semiample Calabi-Yau hypersurfaces (and, more generally, nef complete intersections) are realized as non-nef quasi-smooth complete intersections in higher dimensional toric varieties (with the sum of the degrees of the hypersurfaces anticanonical). Such complete intersections have not been studied so far. It will be nice to have a similar deformation result for them. Because, in this case, it will show that Calabi-Yau complete intersections in toric varieties preserve the property of being complete intersections in toric varieties under complex deformations. As an application to the classification of Calabi-Yau varieties, it will suffice to look for those Calabi-Yau complete intersections in toric varieties that have only "polynomial" deformations, which means that all deformations are realized inside the ambient toric variety. Certainly, we expect the result to hold for singular Calabi-Yau complete intersections as well. This also leads us to the following question: is it true that every Calabi-Yau variety can be realized as a complete intersection in a toric variety? While this may not look believable at the moment with vastly different constructions of Calabi-Yau varieties (e.g., complete intersections in Grassmanians and Flag Manifolds, Voisin-Borcea construction and

1991 Mathematics Subject Classification. Primary: 14M25.

Key words and phrases. Deformations, Calabi-Yau varieties, toric varieties, mirror symmetry. 
the Pfaffian Calabi-Yau manifold), we want to make a prediction of a seemingly achievable goal:

Conjecture 1.1. Extremal transitions (contractions followed by smoothing) of Calabi-Yau complete intersections in toric varieties are again Calabi-Yau complete intersections in toric varieties and such Calabi-Yau varieties are all connected to each other by chains of extremal transitions.

In this conjecture, we assume that the complete intersections of hypersurfaces have the property that the sum of the degrees is anticanonical. In physics, it was expected that the extremal transitions of Calabi-Yau varieties connect them all together into a single web. As a consequence of the above conjecture, Calabi-Yau complete intersections in Grassmanians and Flag Manifolds must be realized as complete intersections in toric varieties, because they degenerate to Calabi-Yau complete intersections in toric varieties. We also want to make another conjecture that Grassmanians and Flag manifolds can be realized as complete intersections in toric varieties. The reason for this is that the deformations of Calabi-Yau hypersurfaces in toric varieties are induced by the deformations of the ambient toric varieties, which have been realized as complete intersections in another toric variety. But it is known that Grassmanians and Flag manifolds are deformations of toric varieties. In particular, describing deformations of (singular) toric varieties is an important problem.

The first example of non-polynomial deformations of a Calabi-Yau hypersurface in $\mathbb{P}(1,1,2,2,2)$, due to Sheldon Katz and David Morrison (see [CadFKMo]), was realized as a $(4,2)$ complete intersection in $\mathbb{P}^{5}$. Even though this example was helpful in creating our general construction, it turned out that there is some flexibility in realizing the deformations of Calabi-Yau hypersurfaces as complete intersections in toric varieties. However, it didn't help to avoid the following technical problem. We cannot make all deformations of a Calabi-Yau hypersurface $X$ simultaneously as complete intersections in one toric variety, but rather we do combine the polynomial deformations together with all those non-polynomial deformations which correspond, by the Kodaira-Spencer map, to a basis in $H^{1}\left(X, \mathcal{T}_{X}\right)$ associated to one of the edges of the reflexive polytope. Despite this technical difficulty, the next priority seems to be in studying the new Calabi-Yau complete intersections achieved in this paper. Since, these complete intersections are non-nef, the cohomology of them will be harder to compute than the cohomology of the nef ones, which in the case of nef nondegenerate intersections follows from [M3] using a "Cayley" trick. Fortunately, the complete intersections in our construction are quasismooth (i.e., V-submanifolds) in simplicial toric varieties, which should help to compute its cohomology.

The plan of our paper is to quickly introduce the reader to the toric varieties and review the results on the infinitesimal deformations of Calabi-Yau hypersurfaces in Section 2. Then, in Section 3, we consider different constructions of "nonpolynomial" complex deformations of Calabi-Yau hypersurfaces which are crepant resolutions of Calabi-Yau hypersurfaces in weighted projective spaces. The last section contains our general construction which realizes the ambient toric variety, the Calabi-Yau hypersurface in it and the non-polynomial deformations as quasismooth complete intersections in a higher dimensional simplicial toric variety. 


\section{Toric VARIETIES, CAlabi-YAU hypersurfaces AND DEFormations.}

This section reviews the definition of a toric variety, and some facts about CalabiYau hypersurfaces. References on toric varieties include [C, F, D, Od].

As usual, let $N$ be a lattice of rank $d$. A fan $\Sigma$ is a finite collection of strongly convex rational polyhedral cones in $N_{\mathbb{R}}$ such that each face of a cone in $\Sigma$ belongs to $\Sigma$ and the intersection of two cones in $\Sigma$ is a face of each.

Assume that 1-dimensional cones of $\Sigma$ span $N_{\mathbb{R}}$. Let $x_{1}, \ldots, x_{n}$ be coordinates on $\mathbb{C}^{n}$, corresponding to the minimal integral generators $e_{1}, \ldots, e_{n}$ of the 1-dimensional cones in $\Sigma$, and let $B(\Sigma)=\left\langle\prod_{e_{i} \notin \sigma} x_{i}: \sigma \in \Sigma\right\rangle$ be the ideal in $\mathbb{C}\left[x_{1}, \ldots, x_{n}\right]$. Consider the complement to the closed subset defined by $B(\Sigma)$, which is a Zariski open set $\mathbb{C}^{n} \backslash \mathbf{V}(B(\Sigma))$. This set is invariant under the diagonal action of an affine algebraic group $G=G(\Sigma)$, a subgroup of $\left(\mathbb{C}^{*}\right)^{n}$, defined by the equations $\prod_{i=1}^{n} x_{i}^{\left\langle m, e_{i}\right\rangle}=1$, for $m \in M=\operatorname{Hom}(N, \mathbb{Z})$, where $\langle$,$\rangle is the pairing. The toric$ variety $\mathbf{P}_{\Sigma}$, associated with the fan $\Sigma$, is the categorical quotient $\left(\mathbb{C}^{n} \backslash \mathbf{V}(B(\Sigma)) / G\right.$. The ring $S:=S(\Sigma)=\mathbb{C}\left[x_{1}, \ldots, x_{n}\right]$ is the homogeneous coordinate ring of $\mathbf{P}_{\Sigma}$ graded by the Chow group $A_{d-1}\left(\mathbf{P}_{\Sigma}\right): \operatorname{deg}\left(\prod_{i=1}^{n} x_{i}^{a_{i}}\right)=\left[\sum_{i=1}^{n} a_{i} D_{i}\right]$, where $D_{i}$ is an irreducible divisor given by $x_{i}=0$.

Semiample anticanonical nondegenerate (transversal to the torus orbits) hypersurfaces in a complete simplicial toric varieties are important examples of CalabiYau varieties. They are defined by the homogeneous polynomials $f \in S_{\beta}$ (here, $\beta=\left[\sum_{i=1}^{n} D_{i}\right]$ is the anticanonical degree) such that $x_{i} \partial f / \partial x_{i}$ do not vanish simultaneously on $\mathbf{P}_{\Sigma}$. The fact that $D=\sum_{i=1}^{n} D_{i}$ is semiample, implies that the associated polytope

$$
\Delta:=\Delta_{D}=\left\{m \in M_{\mathbb{R}}:\left\langle m, e_{i}\right\rangle \geq-1 \text { for all } i\right\} \subset M_{\mathbb{R}}
$$

is reflexive, which means all of its vertices are lattice points, the only interior point is the origin 0 , and the same property has to hold for the dual polytope

$$
\Delta^{\circ}=\left\{n \in N_{\mathbb{R}}:\langle m, n\rangle \geq-1 \text { for all } m \in \Delta\right\} \subset M_{\mathbb{R}} .
$$

Moreover, the minimal integral generators $e_{1}, \ldots, e_{n}$ of the 1-dimensional cones lie on the boundary of $\Delta^{\circ}$. Since $X$ is a big and nef hypersurface, by Proposition 1.2 in [M2], there is the associated toric morphism $\pi: \mathbf{P}_{\Sigma} \rightarrow \mathbf{P}_{\Sigma_{X}}$, where $\Sigma_{X}$ is the fan whose cones are precisely those generated by the faces of the polytope $\Delta^{\circ}$. For instance, a 2-dimensional cone in $\Sigma_{X}$ is a cone over an edge $\Gamma^{*} \subset \Delta^{\circ}$.

When a variety $X$ is smooth, its space of infinitesimal deformations is classified by the first cohomology $H^{1}\left(X, \mathcal{T}_{X}\right)$ with coefficients in the tangent sheaf $\mathcal{T}_{X}$. For semiample anticanonical nondegenerate hypersurfaces $X \subset \mathbf{P}_{\Sigma}$ in a complete simplicial toric variety, there is a natural splitting

$$
H^{1}\left(X, \mathcal{T}_{X}\right)=H_{\text {poly }}^{1}\left(X, \mathcal{T}_{X}\right) \oplus H_{\text {nonp }}^{1}\left(X, \mathcal{T}_{X}\right),
$$

where $H_{\text {poly }}^{1}\left(X, \mathcal{T}_{X}\right)$ is the image of the map $H^{0}\left(X, \mathcal{N}_{X / \mathbf{P}_{\Sigma}}\right) \rightarrow H^{1}\left(X, \mathcal{T}_{X}\right)$, which classifies all deformations of $X$ inside $\mathbf{P}_{\Sigma}$. While the space $H_{\text {nonp }}^{1}\left(X, \mathcal{T}_{X}\right)$ of nonpolynomial infinitesimal deformations of $X$ can be thought as the cokernel of the last map, a natural description is given in Theorem 7.1 in [M4] (also, see [M5, Section 1]).

Proposition 2.1. Let $X \subset \mathbf{P}_{\Sigma}$ be a semiample anticanonical nondegenerate hypersurface in a complete simplicial toric variety, defined by $f \in S_{\beta}$. Then there are 
natural isomorphisms

$$
H_{\text {poly }}^{1}\left(X, \mathcal{T}_{X}\right) \cong R_{1}(f)_{\beta}, \quad H_{\text {nonp }}^{1}\left(X, \mathcal{T}_{X}\right) \cong \bigoplus_{\substack{\sigma \in \Sigma_{X}, \operatorname{dim} \sigma=2 \\ e_{l} \in \operatorname{int}(\sigma)}}\left(S /\left\langle x_{l}\right\rangle\right)_{\beta_{1}^{\sigma}},
$$

where $R_{1}(f)=S / J_{1}(f)$ with $J_{1}(f)=\left\langle x_{1}\left(\partial f / \partial x_{1}\right), \ldots, x_{n}\left(\partial f / \partial x_{n}\right)\right\rangle: x_{1} \cdots x_{n}$, and $\beta_{1}^{\sigma}=\sum_{e_{i} \subset \sigma} \operatorname{deg}\left(x_{i}\right)$.

Corollary 2.2. Let $X$ be a semiample anticanonical nondegenerate hypersurface in a complete simplicial toric variety $\mathbf{P}_{\Sigma}$, such that the minimal lattice generators $e_{1}, \ldots, e_{n}$ of the 1-dimensional cones in $\Sigma$ include all lattice points in the edges of $\Delta^{\circ}$, the dual of the reflexive polytope $\Delta$ associated to the anticanonical divisor. Then

$$
\begin{gathered}
\operatorname{dim} H_{\text {poly }}^{1}\left(X, \mathcal{T}_{X}\right)=l(\Delta)-d-1-\sum_{\operatorname{codim} \Gamma=1} l^{*}(\Gamma), \\
\operatorname{dim} H_{\text {nonp }}^{1}\left(X, \mathcal{T}_{X}\right)=\sum_{\operatorname{codim} \Gamma=2} l^{*}(\Gamma) l^{*}\left(\Gamma^{*}\right),
\end{gathered}
$$

where the sum is by faces $\Gamma$ of $\Delta, \Gamma^{*}$ is the corresponding dual face of $\Delta^{*}$ and $l(\Delta)=\operatorname{Card}(\Delta \cap M)$, while $l^{*}(\Gamma)$ is the number of interior lattice points in $\Gamma$. Moreover, if $e_{1}, \ldots, e_{n}$ contain $\Delta^{\circ} \cap N-\{0\}$, except, possibly, the interior points of the facets of $\Delta^{\circ}$, then $X$ is a minimal Calabi-Yau orbifold.

Proof. The fact that the hypersurface $X$ is a minimal Calabi-Yau orbifold is essentially shown in Proposition 4.1.3 of $[\mathrm{CK}]$. One simply has to note that the singularities of a semiample nondegenerate hypersurface come from singularities of the toric variety, which are all terminal by [CK, Lemma 4.1.2]. Hence, singularities of $X$ are also terminal.

Arguments given in Remark 5.3 of [M2] combined with the description of the space $H^{1}\left(X, \mathcal{T}_{X}\right)$ in Proposition 2.1 produce the required dimension formulas.

We can also determine the polynomial defining a partial crepant resolution of a given Calabi-Yau hypersurface $Y$ in a complete toric variety $\mathbf{P}_{\Sigma^{\prime}}$. Let $Y$ be determined by a homogeneous polynomial

$$
\sum_{m \in \Delta \cap M} a_{m} \prod_{i=1}^{n} z_{i}^{1+\left\langle m, e_{i}^{\prime}\right\rangle}
$$

in $S\left(\Sigma^{\prime}\right)$, where $\Delta$ is the polytope in $M_{\mathbb{R}}$ given by the conditions $\left\langle m, e_{i}^{\prime}\right\rangle \geq-1$ and $e_{i}^{\prime}$ are lattice generators of the 1-dimensional cones in $\Sigma^{\prime}$. Then, if the fan $\Sigma$ is a subdivision of $\Sigma^{\prime}$, the proper transform $X \subset \mathbf{P}_{\Sigma}$ of the hypersurface $Y$ corresponds to

$$
\sum_{m \in \Delta \cap M} a_{m} \prod_{i=1}^{n} x_{i}^{1+\left\langle m, e_{i}\right\rangle}
$$

in $S(\Sigma)$.

\section{Calabi-Yau deformations arising from Weighted Projective spaces.}

First, we will show how our construction works for some examples.

The simplest example of a non-polynomial deformation of a Calabi-Yau hypersurface arises from the weighted projective space $\mathbb{P}(1,1,2,2,2)$. This case, considered in [CadFKMo], was described in details in [M5, Example 2.1]. Let us recall this construction. 
Example 3.1. Consider the quasismooth Calabi-Yau hypersurface $Y$ in $\mathbb{P}(1,1,2,2,2)$ defined by the degree 8 polynomial

$$
z_{1}^{8}+z_{2}^{8}+z_{3}^{4}+z_{4}^{4}+z_{5}^{4}=0 .
$$

The crepant desingularization of $\mathbb{P}(1,1,2,2,2)$ is the toric variety $\mathbf{P}_{\Sigma}$ whose fan has the following integral generators of the 1-dimensional cones:

$$
\{(-1,-2,-2,-2),(1,0,0,0),(0,1,0,0),(0,0,1,0),(0,0,0,1),(0,-1,-1,-1)\} .
$$

If $x_{1}, x_{2}, x_{3}, x_{4}, x_{5}, x_{6}$ denote the homogeneous coordinates of $\mathbf{P}_{\Sigma}$, corresponding to these points, then the crepant resolution $X$ of $Y$ is defined by the polynomial

$$
f(x)=x_{1}^{8} x_{6}^{4}+x_{2}^{8} x_{6}^{4}+x_{3}^{4}+x_{4}^{4}+x_{5}^{4} .
$$

Both the toric variety $\mathbf{P}_{\Sigma}$ and the minimal Calabi-Yau hypersurface $X$ can be embedded into the product $\mathbb{P}^{5} \times \mathbb{P}^{1}$ by sending

$$
\left(x_{1}, x_{2}, x_{3}, x_{4}, x_{5}, x_{6}\right) \mapsto\left(x_{1} x_{2} x_{6}, x_{1}^{2} x_{6}, x_{2}^{2} x_{6}, x_{3}, x_{4}, x_{5}\right) \times\left(x_{1}, x_{2}\right) .
$$

It is straightforward to check that this is an inclusion. If $y_{0}, \ldots, y_{5}$ and $z_{1}, z_{2}$ are the homogeneous coordinates on the 2 factors, the hypersurface $X$ becomes a Calabi-Yau complete intersection given by

$$
y_{1}^{4}+y_{2}^{4}+y_{3}^{4}+y_{4}^{4}+y_{5}^{4}=0, \quad y_{2} z_{1}=y_{0} z_{2}, \quad y_{1} z_{2}=y_{0} z_{1},
$$

where the last two equations determine the variety $\mathbf{P}_{\Sigma}$.

Using the dimension formulas in Proposition 2.2 and a computer program (e.g., see $[\mathrm{KS}])$, one can find that $\operatorname{dim} H_{\text {poly }}^{1}\left(X, \mathcal{T}_{X}\right)=83$ and $\operatorname{dim} H_{\text {nonp }}^{1}\left(X, \mathcal{T}_{X}\right)=3$. Polynomial deformations of the hypersurface $X \subset \mathbf{P}_{\Sigma}$, which leave the hypersurface inside its ambient space $\mathbf{P}_{\Sigma}$, are created by deforming the coefficients of the quartic, while the rest - the non-polynomial deformations - correspond to the family

$$
y_{2} z_{1}=\left(y_{0}+\lambda_{3} y_{3}+\lambda_{4} y_{4}+\lambda_{5} y_{5}\right) z_{2}, \quad y_{1} z_{2}=y_{0} z_{1},
$$

which also induce deformations of the toric variety $\mathbf{P}_{\Sigma}$.

The projection of the non-polynomial deformations of $X$ onto the first factor $\mathbb{P}^{5} \times \mathbb{P}^{1} \rightarrow \mathbb{P}^{5}$ gives the Calabi-Yau complete intersection

$$
y_{1}^{4}+y_{2}^{4}+y_{3}^{4}+y_{4}^{4}+y_{5}^{4}=0, \quad y_{1} y_{2}=y_{0}\left(y_{0}+\lambda_{3} y_{3}+\lambda_{4} y_{4}+\lambda_{5} y_{5}\right),
$$

which is a deformation of the quasismooth hypersurface $Y$ in $\mathbb{P}(1,1,2,2,2)$ : the hypersurface $Y$ together with its ambient space are embedded into $\mathbb{P}^{5}$ by the degree 2 linear system:

$$
\left(z_{1}, z_{2}, z_{3}, z_{4}, z_{5}\right) \mapsto\left(z_{1} z_{2}, z_{1}^{2}, z_{2}^{2}, z_{3}, z_{4}, z_{5}\right) .
$$

Moreover, the Calabi-Yau variety $Y$ can be smoothed to a generic complete intersection of degree 4 and 2 hypersurfaces, and the process of going from $X$ to this smooth Calabi-Yau complete intersection is called an extremal transition (see [CK, Definition 6.2.9.]).

We now make an important observation that the deformation of $Y$ is realized as a complete intersection of 2 hypersurfaces, while $X$ requires 3 . This leads us to the question whether the deformations of $X$ can also be realized as a complete intersection of 2 hypersurfaces, which we are going to answer positively. 
Embed the hypersurface $X$ with its ambient space $\mathbf{P}_{\Sigma}$ into $\mathbb{P}^{4} \times \mathbb{P}^{1}$ using the map

$$
\left(x_{1}, x_{2}, x_{3}, x_{4}, x_{5}, x_{6}\right) \mapsto\left(x_{1}^{2} x_{6}, x_{2}^{2} x_{6}, x_{3}, x_{4}, x_{5}\right) \times\left(x_{1}, x_{2}\right) .
$$

The image of $X$ is a Calabi-Yau complete intersection:

$$
y_{1}^{4}+y_{2}^{4}+y_{3}^{4}+y_{4}^{4}+y_{5}^{4}=0, \quad y_{1} z_{2}^{2}=y_{2} z_{1}^{2},
$$

and the last equation cuts out the subvariety isomorphic to $\mathbf{P}_{\Sigma}$ in $\mathbb{P}^{4} \times \mathbb{P}^{1}$. Then all deformations of $X$ are given by deforming the degree $(4,0)$ hypersurface and by the family

$$
y_{1} z_{2}^{2}=y_{2} z_{1}^{2}+\left(\lambda_{3} y_{3}+\lambda_{4} y_{4}+\lambda_{5} y_{5}\right) z_{1} z_{2}
$$

of degree $(1,2)$ hypersurfaces, which correspond to deformations of the toric variety $\mathbf{P}_{\Sigma}$.

The second example, we consider in this section, is coming from $\mathbb{P}(1,1,2,2,6)$.

Example 3.2. The weighted projective space $\mathbb{P}(1,1,2,2,6)$ has the fan consisting of cones with generators

$$
\{(-1,-2,-2,-6),(1,0,0,0),(0,1,0,0),(0,0,1,0),(0,0,0,1),\} .
$$

The convex hull of these points is a reflexive polytope, such that the only extra lattice points on its boundary are $(0,-1,-1,-3)$ and $(0,0,0,-1)$. Note that subdividing the fan using $(0,-1,-1,-3)$ is enough to get a complete simplicial toric variety $\mathbf{P}_{\Sigma}$ such that the proper transform of a nondegenerate Calabi-Yau hypersurface $Y$ in $\mathbb{P}(1,1,2,2,6)$ is smooth. This is so, since $(0,0,0,-1)$ is in the interior of a 4-dimensional cone, and the nondegenerate hypersurface $Y$ will miss the corresponding singular point of $\mathbb{P}(1,1,2,2,6)$. Let $Y$ be given by the degree 12 polynomial

$$
z_{1}^{12}+z_{2}^{12}+z_{3}^{6}+z_{4}^{6}+z_{5}^{2}=0
$$

where $z_{1}, \ldots, z_{5}$ correspond to the lattice points (3). Then its crepant resolution $X \subset \mathbf{P}_{\Sigma}$ is an anticanonical nondegenerate hypersurface

$$
x_{1}^{12} x_{6}^{6}+x_{2}^{12} x_{6}^{6}+x_{3}^{6}+x_{4}^{6}+x_{5}^{2}=0,
$$

where $x_{1}, \ldots, x_{5}, x_{6}$ correspond to $(3)$ and $(0,-1,-1,-3)$.

In this case, we find $\operatorname{dim} H_{\text {poly }}^{1}\left(X, \mathcal{T}_{X}\right)=126$ and $\operatorname{dim} H_{\text {nonp }}^{1}\left(X, \mathcal{T}_{X}\right)=2$.

Embed the toric variety $\mathbf{P}_{\Sigma}$ and the smooth Calabi-Yau hypersurface $X$ into $\mathbb{P}(1,1,1,1,3) \times \mathbb{P}^{1}$ by the same map as in (2). Then $X$ is isomorphic to a CalabiYau complete intersection in $\mathbb{P}(1,1,1,1,3) \times \mathbb{P}^{1}$ given by

$$
y_{1}^{6}+y_{2}^{6}+y_{3}^{6}+y_{4}^{6}+y_{5}^{2}=0, \quad y_{1} z_{2}^{2}=y_{2} z_{1}^{2},
$$

and the non-polynomial deformations, corresponding to $H_{\text {nonp }}^{1}\left(X, \mathcal{T}_{X}\right)$, are given by replacing the second equation with

$$
y_{1} z_{2}^{2}=y_{2} z_{1}^{2}+\left(\lambda_{3} y_{3}+\lambda_{4} y_{4}\right) z_{1} z_{2} .
$$

Using the above two examples, one can make a prediction that if a minimal semiample Calabi-Yau hypersurface $X$ in a complete simplicial toric variety $\mathbf{P}_{\Sigma}$ is given, then in order to get the deformations the ambient variety $\mathbf{P}_{\Sigma}$ must be embedded into another toric variety with the map given in homogeneous coordinates by

$$
\left(x_{1}, x_{2}, x_{1}^{2} x_{6}, x_{2}^{2} x_{6}, x_{3}, x_{4}, x_{5}, x_{7}, \ldots\right) \text {, }
$$


where $x_{1}, x_{2}, x_{6}$ correspond to lattice points $e_{1}, e_{2}, e_{6}$ such that $e_{6}$ is the middle point between $e_{1}$ and $e_{2}$. It turns out that this will work well as long as there is only one lattice point on the line segment between $e_{1}$ and $e_{2}$. In the next section we will present a general construction, which in the particular situation with $\mathbb{P}(1,1,2,2,2)$ looks as the following.

Example 3.3. Let $X$ be the minimal Calabi-Yau hypersurface defined by

$$
x_{1}^{8} x_{6}^{4}+x_{2}^{8} x_{6}^{4}+x_{3}^{4}+x_{4}^{4}+x_{5}^{4}=0
$$

in the toric variety $\mathbf{P}_{\Sigma}$ as in Example 3.1. Then consider another 5-dimensional toric variety $\mathbf{P}_{\Sigma^{\prime}}$, whose fan $\Sigma^{\prime}$ has the following minimal integral generators along the one-dimensional cones:

$$
\begin{aligned}
\{(1,1,1,1,1),(-1,-2,-2,-2,-1),(0,-1,-1,-1,-1),(0,0,0,0,1), \\
(0,1,0,0,0),(0,0,1,0,0),(0,0,0,1,0)\} .
\end{aligned}
$$

The maximal cones of the fan are spanned by one of the first two generators and four of the last five. By Exercise in [F, page 41], this toric variety is a $\mathbb{P}^{4}$ fiber bundle over $\mathbb{P}^{1}$. Moreover, this is a crepant resolution of a Fano toric variety and the listed generators are the vertices of a reflexive polytope. The toric variety $\mathbf{P}_{\Sigma}$ can be embedded into $\mathbf{P}_{\Sigma^{\prime}}$ by the map

$$
\left(x_{1}, \ldots, x_{6}\right) \mapsto\left(x_{1}, x_{2}, x_{1} x_{6}, x_{2} x_{6}, x_{3}, x_{4}, x_{5}\right),
$$

where components are homogeneous coordinates $s_{1}, t_{1}, s_{2}, t_{0}, y_{3}, y_{4}, y_{5}$ (notation is made similar to the one in the next section), corresponding to the above lattice points. Then the image of $\mathbf{P}_{\Sigma}$ is a hypersurface given by $s_{1} t_{0}=s_{2} t_{1}$, and the Calabi-Yau hypersurface $X$ is now a complete intersection of $s_{1} t_{0}=s_{2} t_{1}$ with $s_{1}^{4} s_{2}^{4}+t_{0}^{4} t_{1}^{4}+y_{3}^{4}+y_{4}^{4}+y_{5}^{4}$. The non-polynomial deformations are represented by the family of complete intersections:

$$
s_{1} t_{0}=s_{2} t_{1}+\lambda_{3} y_{3}+\lambda_{4} y_{4}+\lambda_{5} y_{5} \quad s_{1}^{4} s_{2}^{4}+t_{0}^{4} t_{1}^{4}+y_{3}^{4}+y_{4}^{4}+y_{5}^{4}
$$

with three parameters $\lambda_{3}, \lambda_{4}$ and $\lambda_{5}$.

\section{Embedding of CAlabi-Yau Deformations Using one EDGE.}

First, we consider a simpler construction. Here is the idea of how we achieved it. Using the examples from the previous section, we guess what should be the map that embeds the toric variety with its Calabi-Yau hypersurface into another toric variety. A problem is then to find the combinatorial data that would produce a toric variety compatible with the map.

While we explained how the construction was found, it is more convenient to give the answer for the new ambient space first. Let $\mathbf{P}_{\Sigma}$ be a complete simplicial toric variety with a semiample anticanonical degree, such that the minimal lattice generators $e_{1}, \ldots, e_{n}$ of the 1-dimensional cones in $\Sigma$ include all lattice points in one of the edges $\Gamma^{*}$ of $\Delta^{\circ}$. We order the latter lattice points $e_{i_{0}}, e_{i_{1}}, \ldots, e_{i_{k+1}}$ as in the following picture, where the cones of $\Sigma$ subdivide a 2-dimensional cone over $\Gamma^{*}$.

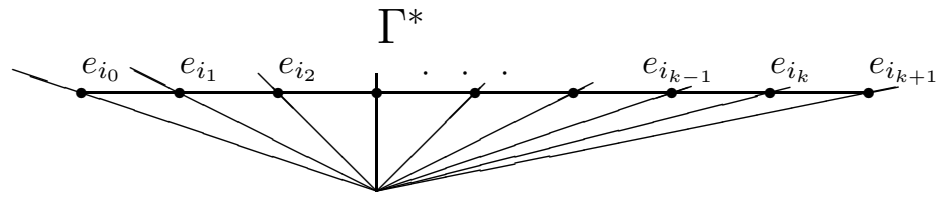


We want to embed $\mathbf{P}_{\Sigma}$ into another toric variety, which is denoted $\mathbf{P}_{\Sigma\left(\Gamma^{*}\right)}$. The new toric variety must have a representation as a quotient

$$
\mathbf{P}_{\Sigma\left(\Gamma^{*}\right)} \cong\left(\mathbb{C}^{n+k} \backslash \mathbf{V}\left(B\left(\Sigma\left(\Gamma^{*}\right)\right)\right) / G\left(\Sigma\left(\Gamma^{*}\right)\right)\right.
$$

for some fan $\Sigma\left(\Gamma^{*}\right)$. Our goal is to determine this fan. Using the example from the previous section, we first guess that the embedding $\phi_{\Gamma^{*}}: \mathbf{P}_{\Sigma} \rightarrow \mathbf{P}_{\Sigma\left(\Gamma^{*}\right)}$ can be written as follows. If $x=\left(x_{1}, \ldots, x_{n}\right)$ represents a point in $\mathbf{P}_{\Sigma}$, then $\phi_{\Gamma^{*}}(x)$ is the point represented by the homogeneous coordinates of $\mathbf{P}_{\Sigma\left(\Gamma^{*}\right)}$ :

$$
\begin{aligned}
& s_{j}=x_{i_{0}} x_{i_{1}} \ldots x_{i_{j-2}} x_{i_{j-1}}, \quad \text { for } j=1, \ldots, k+1, \\
& \quad t_{j}=x_{i_{j+1}} x_{i_{j+2}} \ldots x_{i_{k}} x_{i_{k+1}}, \quad \text { for } j=0, \ldots, k, \text { and } y_{i}=x_{i} \text {, for } e_{i} \notin \Gamma^{*},
\end{aligned}
$$

where the coordinates $s_{j}, t_{j}$ correspond to the unknown lattice points $\tilde{e}_{s_{j}}, \tilde{e}_{t_{j}}$ in the lattice $N \oplus \mathbb{Z}^{k}$, and $y_{i}$ correspond to $\tilde{e}_{i}=e_{i}$ in $N \oplus \mathbb{Z}^{k}$ for $e_{i} \notin \Gamma^{*}$. So, the fan $\Sigma\left(\Gamma^{*}\right)$ must lie in the lattice $N \oplus \mathbb{Z}^{k}$ with the minimal integral generators of the 1-dimensional cones: $\tilde{e}_{s_{1}}, \ldots, \tilde{e}_{s_{k+1}}, \tilde{e}_{t_{0}}, \ldots, \tilde{e}_{t_{k}}$ and $\tilde{e}_{i}$ for $e_{i} \notin \Gamma^{*}$. Instead of explaining how to find the unknown lattice points, we will rather give an answer right away. The idea of deriving these lattice points is to use the fact that the map $\phi_{\Gamma^{*}}(x)$ has to be equivariant with respect to the group actions and it will appear in the proof of the theorem below. Let $v_{1}, \ldots, v_{k}$ be a standard basis of $\mathbb{Z}^{k}$, then set

$$
\begin{aligned}
& \tilde{e}_{s_{j}}=v_{j}, \text { for } j=1, \ldots, k, \\
& \tilde{e}_{s_{k+1}}=e_{i_{0}}-v_{1}-\cdots-v_{k}, \\
& \tilde{e}_{t_{j-1}}=v_{j}+\frac{1}{k+1}\left(e_{i_{k+1}}-e_{i_{0}}\right), \text { for } j=1, \ldots, k, \\
& \tilde{e}_{t_{k}}=e_{i_{0}}-v_{1}-\cdots-v_{k}+\frac{1}{k+1}\left(e_{i_{k+1}}-e_{i_{0}}\right) .
\end{aligned}
$$

Next, form a collection $\Sigma\left(\Gamma^{*}\right)$ of cones in $N_{\mathbb{R}} \oplus \mathbb{R}^{k}$, consisting of the cones with their faces of the following types:

(i) $\sigma \in \Sigma$ if $\sigma \cap \Gamma^{*}=\emptyset$,

(ii) $\left\langle e_{i} \in \sigma \backslash\left\{e_{i_{j}}\right\}, \tilde{e}_{t_{0}}, \tilde{e}_{t_{1}}, \ldots, \tilde{e}_{t_{j-1}}, \tilde{e}_{s_{j+1}}, \ldots, \tilde{e}_{s_{k+1}}\right\rangle$ if $\sigma \cap \Gamma^{*}=\left\{e_{i_{j}}\right\}$,

(iii) $\left\langle e_{i} \in \sigma \backslash\left(\sigma \cap \Gamma^{*}\right), \tilde{e}_{t_{0}}, \tilde{e}_{t_{1}}, \ldots, \tilde{e}_{t_{j}}, \tilde{e}_{s_{j+1}}, \ldots, \tilde{e}_{s_{k+1}}\right\rangle$ if $\sigma \cap \Gamma^{*}=\left\{e_{i_{j}}, e_{i_{j+1}}\right\}$,

where we indicated the generators of the cones and $e_{i}$ run by all possible generators in the complements. To get this collection of cones note which variables vanish in (4) if $x_{i_{j}}=0$. Notice also that the cones in (i) do not have the full dimension of the space $N_{\mathbb{R}} \oplus \mathbb{R}^{k}$. So, the above cones do not necessarily cover the whole space. Magically, the collection $\Sigma\left(\Gamma^{*}\right)$ is a fan.

Lemma 4.1. The collection of cones $\Sigma\left(\Gamma^{*}\right)$ forms a simplicial fan in the lattice $N \oplus \mathbb{Z}^{k}$.

Proof. The fact that $\Sigma\left(\Gamma^{*}\right)$ is simplicial follows from an easy checking that the generators of the cones of types (ii)-(iii) are linearly independent and the condition that $\Sigma$ was simplicial. We need to show that the cones in $\Sigma\left(\Gamma^{*}\right)$ meet along their faces, or, in other words, that the intersection of the cones lies in the face generated by the common minimal lattice generators.

Observe the property satisfied by the cones in $\Sigma\left(\Gamma^{*}\right)$ : if $\sigma^{\prime}$ denotes the cone in (ii)-(iii) constructed from $\sigma \in \Sigma$, then the intersection of $\sigma^{\prime}$ with the subspace $N_{\mathbb{R}}$ 
in $N_{\mathbb{R}} \oplus \mathbb{R}^{k}$ is exactly $\sigma$. Indeed, for $\sigma^{\prime}$ of type (ii), if

$\alpha_{t_{0}} \tilde{e}_{t_{0}}+\cdots+\alpha_{t_{j-1}} \tilde{e}_{t_{j-1}}+\alpha_{s_{j+1}} \tilde{e}_{s_{j+1}}+\cdots+\alpha_{s_{k+1}} \tilde{e}_{s_{k+1}}+\sum_{e_{i} \in \sigma \backslash\left\{e_{i_{j}}\right\}} \alpha_{i} e_{i} \in N_{\mathbb{R}} \oplus \mathbf{0}$,

where all the coefficients $\alpha \geq 0$, then, by comparing the coefficients at the basis vectors $v_{1}, \ldots, v_{k}$, we deduce

$$
\alpha_{t_{0}}=\cdots=\alpha_{t_{j-1}}=\alpha_{s_{j+1}}=\cdots \alpha_{s_{k+1}} .
$$

But in this case, the above sum is

$$
\alpha_{s_{k+1}}\left(\tilde{e}_{i_{0}}+\frac{j}{k+1}\left(e_{i_{k+1}}-e_{i_{0}}\right)\right)+\sum_{e_{i} \in \sigma \backslash\left\{e_{i_{j}}\right\}} \alpha_{i} e_{i}=\alpha_{s_{k+1}} e_{i_{j}}+\sum_{e_{i} \in \sigma \backslash\left\{e_{i_{j}}\right\}} \alpha_{i} e_{i},
$$

which belongs to $\sigma$. The other inclusion follows from the equality

$$
e_{i_{j}}=\tilde{e}_{t_{0}}+\cdots+\tilde{e}_{t_{j-1}}+\tilde{e}_{s_{j+1}}+\cdots+\tilde{e}_{s_{k+1}} .
$$

Thus $\sigma^{\prime} \cap N_{\mathbb{R}} \oplus \mathbf{0}=\sigma \oplus \mathbf{0}$. Verification of this property for type (iii) cones is similar.

Now, we can see that the intersection of type (i) cone $\sigma_{1} \oplus \mathbf{0}$ with a cone $\sigma_{2}^{\prime}$ of type (ii) or (iii) is exactly $\left(\sigma_{1} \cap \sigma_{2}\right) \oplus \mathbf{0}$, which is a face of each of the cones.

Next, let us show that the intersection of two distinct type (ii) cones $\sigma_{1}^{\prime}$ and $\sigma_{2}^{\prime}$ is a face of each. A point lying in the intersection would produce the following equality:

$$
\begin{aligned}
& \alpha_{t_{0}} \tilde{e}_{t_{0}}+\cdots+\alpha_{t_{j-1}} \tilde{e}_{t_{j-1}}+\alpha_{s_{j+1}} \tilde{e}_{s_{j+1}}+\cdots+\alpha_{s_{k+1}} \tilde{e}_{s_{k+1}}+\sum_{e_{i} \in \sigma_{1} \backslash\left\{e_{i_{j}}\right\}} \alpha_{i} e_{i} \\
& =\beta_{t_{0}} \tilde{e}_{t_{0}}+\cdots+\beta_{t_{l-1}} \tilde{e}_{t_{l-1}}+\beta_{s_{l+1}} \tilde{e}_{s_{l+1}}+\cdots+\alpha_{s_{k+1}} \tilde{e}_{s_{k+1}}+\sum_{e_{i} \in \sigma_{2} \backslash\left\{e_{i_{l}}\right\}} \alpha_{i} e_{i},
\end{aligned}
$$

where $\sigma_{1} \cap \Gamma^{*}=\left\{e_{i_{j}}\right\}, \sigma_{2} \cap \Gamma^{*}=\left\{e_{i_{l}}\right\}$ and $j \leq l$. Again, comparing coefficients at $v_{1}, \ldots, v_{k}$, we get

$$
\begin{aligned}
& \alpha_{t_{a}}-\alpha_{s_{k+1}}=\beta_{t_{a}}-\beta_{s_{k+1}}, \quad a=0, \ldots, j-1, \\
& \alpha_{s_{a+1}}-\alpha_{s_{k+1}}=\beta_{t_{a}}-\beta_{s_{k+1}}, \quad a=j, \ldots, l-1, \\
& \alpha_{s_{a+1}}-\alpha_{s_{k+1}}=\beta_{s_{a+1}}-\beta_{s_{k+1}}, \quad a=l, \ldots, k .
\end{aligned}
$$

Using this and simplifying the above equality, we have

$$
\begin{aligned}
& \alpha_{s_{k+1}} e_{i_{j}}+\sum_{e_{i} \in \sigma_{1} \backslash\left\{e_{i_{j}}\right\}} \alpha_{i} e_{i} \\
= & \left(\beta_{t_{j}}+\cdots+\beta_{t_{l-1}}-(l-j) \beta_{s_{k+1}}\right) \frac{1}{k+1}\left(e_{i_{k+1}}-e_{i_{0}}\right)+\beta_{s_{k+1}} e_{i_{l}}+\sum_{e_{i} \in \sigma_{2} \backslash\left\{e_{i_{l}}\right\}} \alpha_{i} e_{i} .
\end{aligned}
$$

If $j=l$, then we are done with this case. If $j<l$, consider first the case that both $\alpha_{s_{k+1}}$ and $\beta_{s_{k+1}}$ are not zero. Then note that there exists a point $p=(1-\varepsilon) e_{i_{j}}+\varepsilon e_{i_{l}}$, $0 \leq \varepsilon \leq 1$, between $e_{i_{j}}$ and $e_{i_{l}}$ such that the intersection of the cones $\left\langle p, e_{i} \in\right.$ $\left.\sigma_{1} \backslash\left\{e_{i_{j}}\right\}\right\rangle$ and $\left\langle p, e_{i} \in \sigma_{2} \backslash\left\{e_{i_{l}}\right\}\right\rangle$ is at most the cone generated by $p$ and $\sigma_{1} \cap \sigma_{2}$. We can rewrite the last equality as

$$
\alpha_{s_{k+1}} e_{i_{j}}-\varepsilon\left(\alpha_{s_{j+1}}+\cdots+\alpha_{s_{l}}-(l-j) \alpha_{s_{k+1}}\right) \frac{1}{k+1}\left(e_{i_{k+1}}-e_{i_{0}}\right)+\sum_{e_{i} \in \sigma_{1} \backslash\left\{e_{i_{j}}\right\}} \alpha_{i} e_{i}
$$




$$
=(1-\varepsilon)\left(\beta_{t_{j}}+\cdots+\beta_{t_{l-1}}-(l-j) \beta_{s_{k+1}}\right) \frac{1}{k+1}\left(e_{i_{k+1}}-e_{i_{0}}\right)+\beta_{s_{k+1}} e_{i_{l}}+\sum_{e_{i} \in \sigma_{2} \backslash\left\{e_{i_{l}}\right\}} \alpha_{i} e_{i},
$$

or

$$
\begin{aligned}
\alpha_{s_{k+1}} p- & \varepsilon\left(\alpha_{s_{j+1}}+\cdots+\alpha_{s_{l}}\right) \frac{1}{k+1}\left(e_{i_{k+1}}-e_{i_{0}}\right)+\sum_{e_{i} \in \sigma_{1} \backslash\left\{e_{i_{j}}\right\}} \alpha_{i} e_{i} \\
= & \beta_{s_{k+1}} p+(1-\varepsilon)\left(\beta_{t_{j}}+\cdots+\beta_{t_{l-1}}\right) \frac{1}{k+1}\left(e_{i_{k+1}}-e_{i_{0}}\right) \sum_{e_{i} \in \sigma_{2} \backslash\left\{e_{i_{l}}\right\}} \alpha_{i} e_{i} .
\end{aligned}
$$

Assuming that $\alpha_{s_{k+1}}, \beta_{s_{k+1}} \neq 0$, the above linear combinations would represent a point in the intersection of two convex simplicial cones whose generators are at a point in the plane passing through the origin and $\Gamma^{*}$ and at $e_{i}$ in $\sigma_{1} \backslash\left\{e_{i_{j}}\right\}$ and in $\sigma_{2} \backslash\left\{e_{i_{l}}\right\}$, respectively. By the choice of $p$, we conclude that

$$
\alpha_{s_{j+1}}=\cdots=\alpha_{s_{l}}=\beta_{t_{j}}=\cdots=t_{l-1} .
$$

Hence, $\alpha_{s_{k+1}}=\beta_{s_{k+1}}$ as well, and we can deduce that the intersection $\sigma_{1}^{\prime} \cap \sigma_{2}^{\prime}$ lies in the face generated by the common minimal lattice generators. In the case when one of $\alpha_{s_{k+1}}$ and $\beta_{s_{k+1}}$ vanishes we can assume, by symmetry, that $\beta_{s_{k+1}}=0$. This will lead to an equation where on the left hand side there is point from $\sigma_{1}$ and on the right side - a point from a facet of $\sigma_{2}$ shifted away from $\sigma_{1}$ by the vector $\left(\beta_{t_{j}}+\cdots+\beta_{t_{l-1}}\right)\left(e_{i_{k+1}}-e_{i_{0}}\right)$. This implies $\beta_{t_{j}}=\cdots=\beta_{t_{l-1}}=\alpha_{s_{k+1}}=0$, whence $\alpha_{s_{j+1}}=\cdots=\alpha_{s_{l}}=0$ as well. Thus, $\sigma_{1}^{\prime} \cap \sigma_{2}^{\prime}$ is a face of both $\sigma_{1}^{\prime}$ and $\sigma_{2}^{\prime}$, when these two cones are of type (ii). The other cases of intersections of two cones of type (ii) and (iii) can be shown to hold the required property in a similar manner, and left to the reader.

Here is our first main result which shows that the toric variety $\mathbf{P}_{\Sigma}$ is realized as a complete intersection by the map $\phi_{\Gamma^{*}}$.

Theorem 4.2. Let $\mathbf{P}_{\Sigma}$ be a complete simplicial toric variety with a semiample anticanonical degree, such that the minimal lattice generators $e_{1}, \ldots, e_{n}$ of $\Sigma(1)$ lie on the boundary of a reflexive polytope $\Delta^{\circ}$ and include all lattice points in one of the edges $\Gamma^{*} \subset \Delta^{\circ}$. Then the morphism

$$
\phi_{\Gamma^{*}}: \mathbf{P}_{\Sigma} \rightarrow \mathbf{P}_{\Sigma\left(\Gamma^{*}\right)}
$$

(see (4)) is a well defined embedding. Moreover, the image of $\mathbf{P}_{\Sigma}$ is a quasismooth complete intersection in $\mathbf{P}_{\Sigma\left(\Gamma^{*}\right)}$ given by the equations

$$
s_{j+1} t_{j}=s_{j} t_{j-1}, \text { for } j=1, \ldots, k .
$$

Proof. Recall from Section 2 that the simplicial toric variety $\mathbf{P}_{\Sigma}$ is the geometric quotient of $\mathbb{C}^{n} \backslash \mathbf{V}(B(\Sigma))$ by $G(\Sigma)$. Similarly,

$$
\mathbf{P}_{\Sigma\left(\Gamma^{*}\right)} \cong\left(\mathbb{C}^{n+k} \backslash \mathbf{V}\left(B\left(\Sigma\left(\Gamma^{*}\right)\right)\right) / G\left(\Sigma\left(\Gamma^{*}\right)\right)\right.
$$

We need to show that the map given by (4) is well defined, i.e., given $\left(x_{1}, \ldots, x_{n}\right) \in$ $G(\Sigma)$, then $\phi_{\Gamma^{*}}\left(x_{1}, \ldots, x_{n}\right) \in G\left(\Sigma\left(\Gamma^{*}\right)\right)$, and, also, the complement of $\mathbf{V}(B(\Sigma))$ maps to the complement of $\mathbf{V}\left(B\left(\Sigma\left(\Gamma^{*}\right)\right)\right.$. Take $\left(x_{1}, \ldots, x_{n}\right)$ such that $\prod_{i=1}^{n} x_{i}^{\left\langle m, e_{i}\right\rangle}=$ 
1 , for all $m \in M=\operatorname{Hom}(N, \mathbb{Z})$. Then we must check that $\phi_{\Gamma^{*}}\left(x_{1}, \ldots, x_{n}\right)$ satisfies the equations

$$
\prod_{e_{i} \notin \Gamma^{*}} y_{i}^{\left\langle\tilde{m}, \tilde{e}_{i}\right\rangle} \prod_{j=1}^{k+1} s_{j}^{\left\langle\tilde{m}, \tilde{e}_{s_{j}}\right\rangle} t_{j-1}^{\left\langle\tilde{m}, \tilde{e}_{t_{j-1}}\right\rangle}=1
$$

for all $\tilde{m} \in \operatorname{Hom}\left(N \oplus \mathbb{Z}\left\{v_{1}, \ldots, v_{k}\right\}, \mathbb{Z}\right)$. It is enough to consider two cases: $\tilde{m}$ vanishes on $v_{1}, \ldots, v_{k}$ or $N$. In the first case, substituting (4) into the last equation, we get

$$
\begin{aligned}
& \left(\prod_{e_{i} \notin \Gamma^{*}} x_{i}^{\left\langle\tilde{m}, e_{i}\right\rangle}\right)\left(x_{i_{0}} \cdots x_{i_{k}}\right)^{\left\langle\tilde{m}, e_{i_{0}}\right\rangle}\left(\prod_{j=1}^{k+1}\left(x_{i_{j}} \cdots x_{i_{k+1}}\right)^{\left\langle\tilde{m}, \frac{e_{i_{k+1}}-e_{i_{0}}}{k+1}\right\rangle}\right) x_{i_{k+1}}^{\left\langle\tilde{m}, e_{i_{0}}\right\rangle} \\
& =\prod_{e_{i} \notin \Gamma^{*}} x_{i}^{\left\langle\tilde{m}, e_{i}\right\rangle} \prod_{j=0}^{k+1} x_{i_{j}}^{\left\langle\tilde{m}, e_{i_{0}}+\frac{j}{k+1}\left(e_{i_{k+1}}-e_{i_{0}}\right)\right\rangle}=\prod_{e_{i} \notin \Gamma^{*}} x_{i}^{\left\langle\tilde{m}, e_{i}\right\rangle} \prod_{j=0}^{k+1} x_{i_{j}}^{\left\langle\tilde{m}, e_{i_{j}}\right\rangle} \\
& =\prod_{i=1}^{n} x_{i}^{\left\langle\tilde{m}, e_{i}\right\rangle}=1,
\end{aligned}
$$

because $\tilde{m}$ is a homomorphism from $N$ to $\mathbb{Z}$. For a later use notice that this calculation also shows: if $\phi_{\Gamma^{*}}\left(x_{1}, \ldots, x_{n}\right) \in G\left(\Sigma\left(\Gamma^{*}\right)\right)$, then $\left(x_{1}, \ldots, x_{n}\right) \in G(\Sigma)$. In the second case, when $\tilde{m}$ is zero on $N$, the left-hand side of (6) becomes

$$
\begin{aligned}
& \left(\prod_{j=1}^{k}\left(x_{i_{0}} \cdots x_{i_{j-1}}\right)^{\left\langle\tilde{m}, v_{j}\right\rangle}\right)\left(x_{i_{0}} \cdots x_{i_{k}} \cdot x_{i_{k+1}}\right)^{\left\langle\tilde{m},-v_{1}-\cdots-v_{k}\right\rangle}\left(\prod_{j=1}^{k}\left(x_{i_{j}} \cdots x_{i_{k+1}}\right)^{\left\langle\tilde{m}, v_{j}\right\rangle}\right) \\
& =\left(\prod_{j=1}^{k}\left(x_{i_{0}} \cdots x_{i_{k+1}}\right)^{\left\langle\tilde{m}, v_{j}\right\rangle}\right)\left(x_{i_{0}} \cdots x_{i_{k+1}}\right)^{\left\langle\tilde{m},-v_{1}-\cdots-v_{k}\right\rangle}=1 .
\end{aligned}
$$

Now, suppose that $\left(x_{1}, \ldots, x_{n}\right) \in \mathbb{C}^{n} \backslash \mathbf{V}(B(\Sigma))$, then $\prod_{e_{i} \notin \sigma} x_{i} \neq 0$ for some $\sigma \in \Sigma$. If $\sigma$ does not intersect the edge $\Gamma^{*}$, then $\sigma \in \Sigma\left(\Gamma^{*}\right)$, and, by the construction of $\phi_{\Gamma^{*}}$, all variables corresponding to the generators in $\Sigma\left(\Gamma^{*}\right)$ outside $\sigma$ don't vanish, whence so does the product of them. If $\sigma$ intersects $\Gamma^{*}$, then, by the construction of $\phi_{\Gamma^{*}}$ and of the corresponding cone $\sigma^{\prime} \in \Sigma\left(\Gamma^{*}\right)$ of type (ii)-(iii), the product of the variables for generators outside $\sigma^{\prime}$ will not vanish, so that $\phi_{\Gamma^{*}}(x)$ belongs to the complement of $\mathbf{V}\left(B\left(\Sigma\left(\Gamma^{*}\right)\right.\right.$ in $\mathbb{C}^{n+k}$ This finishes the proof that the map $\phi_{\Gamma^{*}}$ is well defined.

To prove injectivity of $\phi_{\Gamma^{*}}$ one needs to recover all $x_{i}$ from given $s_{1}, \ldots, s_{k+1}$, $t_{0}, \ldots, t_{k}$, and $y_{i}$, accounting for the group actions which represent the toric varieties as geometric quotients. In other words, the "upstairs" map

$$
\phi_{\Gamma^{*}}: \mathbb{C}^{n} \backslash \mathbf{V}(B(\Sigma)) \rightarrow \mathbb{C}^{n+k} \backslash \mathbf{V}\left(B\left(\Sigma\left(\Gamma^{*}\right)\right)\right.
$$

must be one-to-one, and $\phi_{\Gamma^{*}}(G(\Sigma))=G\left(\Sigma\left(\Gamma^{*}\right)\right)$. Note that

$$
x_{i_{j}}=\frac{s_{j+1}}{s_{j}}=\frac{t_{j-1}}{t_{j}}, \text { for } j=1, \ldots, k, \quad x_{i_{0}}=s_{1}, x_{i_{k+1}}=t_{k}, \text { and } x_{i}=y_{i}
$$

for $e_{i} \notin \Gamma^{*}$. Hence, $\phi_{\Gamma^{*}}$ is injective on $\mathbb{C}^{n} \backslash \mathbf{V}(B(\Sigma))$, because $s_{j}$ and $t_{j}$ don't vanish simultaneously by the properties of toric varieties: they correspond to the generators $\tilde{e}_{s_{j}}$ and $\tilde{e}_{t_{j}}$ which do not lie in the same cone of the fan $\Sigma\left(\Gamma^{*}\right)$, by the construction. To show the equality take a point from $G\left(\Sigma\left(\Gamma^{*}\right)\right)$ with homogeneous coordinates $s_{1}, \ldots, s_{k+1}, t_{0}, \ldots, t_{k}$ and $y_{i}$, for $e_{i} \notin \Gamma^{*}$. Then, taking $\tilde{m}$ in (6) such 
that $\tilde{m}$ vanishes on $N$ and $\left\langle\tilde{m}, v_{j}\right\rangle=-1,\left\langle\tilde{m}, v_{j+1}\right\rangle=1,\left\langle\tilde{m}, v_{l}\right\rangle=0$, for $l \neq j, j+1$, we get the equations

$$
s_{j}^{-1} s_{j+1} t_{j-1}^{-1} t_{j}=1, \text { for } j=1, \ldots, k-1 .
$$

Similarly, if $\tilde{m}$ is zero on $N$ and on $v_{1} \ldots, v_{k-1}$, and $\left\langle\tilde{m}, v_{k}\right\rangle=-1$, then (6) becomes $s_{k}^{-1} s_{k+1} t_{k-1}^{-1} t_{k}=1$. Because of (7), these equations show that every point in $G\left(\Sigma\left(\Gamma^{*}\right)\right)$ is in the image of $\phi_{\Gamma^{*}}$. Combining this with an earlier note in the proof that the intersection of the image of $\phi_{\Gamma^{*}}$ with $G\left(\Sigma\left(\Gamma^{*}\right)\right)$ is already in the image of $G(\Sigma)$, we get the equality $\phi_{\Gamma^{*}}(G(\Sigma))=G\left(\Sigma\left(\Gamma^{*}\right)\right)$.

Next, we need to verify that $\phi_{\Gamma^{*}}\left(\mathbf{P}_{\Sigma}\right)$ coincides with the complete intersection $s_{j+1} t_{j}=s_{j} t_{j-1}$, for $j=1, \ldots, k$. One direction is easy by checking that these equations hold for the points in (4). Conversely, suppose that a point in $\mathbf{P}_{\Sigma\left(\Gamma^{*}\right)}$ with homogeneous coordinates $s_{1}, \ldots, s_{k+1}, t_{0}, \ldots, t_{k}$, and $y_{i}$ satisfies the above equations. Then we can determine $\left(x_{1}, \ldots, x_{n}\right)$ uniquely using the equations (7). The problem is whether $\phi_{\Gamma^{*}}\left(x_{1}, \ldots, x_{n}\right)$ gives us back the original $s_{1}, \ldots, s_{k+1}$, $t_{0}, \ldots, t_{k}$, and $y_{i}$. If all $s$ and $t$ are not equal to zero, then the previous paragraph has already showed this. So, let us assume that one of $t$, for instance, is equal to zero and let $k+1 \geq l \geq 1$ be the minimal integer for which $t_{l} \neq 0$ and $t_{0}=\cdots=t_{l-1}=0$. Then notice that if $t_{j-1} \neq 0$ then $t_{j} \neq 0$ as well. Indeed, we have equation $s_{j+1} t_{j}=s_{j} t_{j-1}$. Since $s_{j}$ and $t_{j}$ don't vanish simultaneously, we get a contradiction if $t_{j-1} \neq 0$ and $t_{j}=0$. So, we can assume that $t_{0}=\cdots=t_{l-1}=0$ and $t_{j} \neq 0$ for $j \geq l$. Also, then $s_{j} \neq 0$ for $j \leq l-1$. Equation $s_{l+1} t_{l}=s_{l} t_{l-1}$ implies that $s_{l+1}=0$ since $t_{l} \neq 0$ and $t_{l-1}=0$. Similarly to what we did with $t_{j}$, we conclude that $s_{j}=0$ for $j \geq l+1$. Now, plugging $x_{i_{j}}=t_{j-1} / t_{j}$, for $j=l, \ldots, k$, and $x_{i_{k+1}}=t_{k}$ into $t_{j}=x_{i_{j+1}} x_{i_{j+2}} \ldots x_{i_{k}} x_{i_{k+1}}$ we recover $t_{j}$ for $j \geq l-1$. Doing the same for $s_{j}=x_{i_{0}} x_{i_{1}} \ldots x_{i_{j-2}} x_{i_{j-1}}$ with $x_{i_{j}}=s_{j+1} / s_{j}$ and $x_{i_{0}}=s_{1}$ we get $s_{j}$ for $j \leq l$. Since $x_{i_{l}}=t_{l-1} / t_{l}=0 / t_{l}=0$, equations (4) give us $s_{j}=0$ for $j \geq l+1$ and $t_{j}=0$ for $j \leq l-1$. Thus, each point in the complete intersection is in the image $\phi_{\Gamma^{*}}\left(\mathbf{P}_{\Sigma}\right)$.

To finish the proof we are left to show that the complete intersection $s_{j+1} t_{j}=$ $s_{j} t_{j-1}$, for $j=1, \ldots, k$, is quasismooth. Quasismooth complete intersections in toric varieties were introduced in [M1, Definition 1.1] and are equivalent to $V$ submanifolds (suborbifolds; see $[\mathrm{BC}]$ ) of the ambient toric variety. Recall that a complete intersection defined by the homogeneous polynomials $f_{1}, \ldots, f_{s}$ is quasismooth if the Jacobian matrix $\left(\frac{\partial f_{j}}{\partial x_{i}}(x)\right)_{i, j}$ has maximal rank on the intersection. In our case the Jacobian matrix is

$$
\left(\begin{array}{cccccccccccccc}
t_{0} & -t_{1} & 0 & 0 & \cdots & 0 & s_{1} & -s_{2} & 0 & 0 & \cdots & 0 & 0 & \cdots \\
0 & t_{1} & -t_{2} & 0 & \cdots & 0 & 0 & s_{2} & -s_{3} & 0 & \cdots & 0 & 0 & \cdots \\
0 & 0 & t_{2} & -t_{3} & \cdots & 0 & 0 & 0 & s_{3} & -s_{4} & \cdots & 0 & 0 & \cdots \\
\vdots & & & \ddots & \ddots & \vdots & \vdots & & & \ddots & \ddots & \vdots & 0 & \cdots \\
0 & 0 & 0 & \cdots & t_{k-1} & -t_{k} & 0 & 0 & 0 & \cdots & s_{k} & -s_{k+1} & 0 & \cdots
\end{array}\right)
$$

where rows correspond to the partial derivatives of the defining polynomials $s_{j+1} t_{j}-$ $s_{j} t_{j-1}, j=1, \ldots, k$, with respect to the variables $s_{1}, \ldots, s_{k+1}, t_{0}, \ldots, t_{k}$ and $y_{i}$ for $e_{i} \notin \Gamma^{*}$. As in the previous paragraph, we can assume that a point on the complete intersection will have coordinates $s_{j} \neq 0$ for $j \leq l-1$ and $t_{j} \neq 0$ for $j \geq l$ for some $0 \leq l \leq k+1$. Then it is easy to see that the above matrix will have the maximal rank equal to $k$. The proof of the theorem is complete. 
Remark 4.3. If $X$ is an anticanonical hypersurface defined by a homogeneous polynomial

$$
f(x)=\sum_{m \in \Delta \cap M} a_{m} \prod_{i=1}^{n} x_{i}^{1+\left\langle m, e_{i}\right\rangle}
$$

in the toric variety $\mathbf{P}_{\Sigma}$ satisfying the condition of Theorem 4.2 , then by the map $\phi_{\Gamma^{*}}$ the variety $X$ is isomorphic to a complete intersection in $\mathbf{P}_{\Sigma\left(\Gamma^{*}\right)}$ given by

$$
s_{j+1} t_{j}=s_{j} t_{j-1}, \text { for } j=1, \ldots, k,
$$

and

$$
\sum_{m \in \Delta \cap M} a_{m} X_{m} \prod_{e_{i} \notin \Gamma^{*}} y_{i}^{1+\left\langle m, e_{i}\right\rangle}=0
$$

where

$$
X_{m}=\left\{\begin{array}{ccc}
\left(s_{1} t_{0}\right)^{1+\left\langle m, e_{i_{0}}\right\rangle}\left(t_{0} \cdots t_{k}\right)^{\left\langle m, e_{i_{1}}-e_{i_{0}}\right\rangle} & \text { if } & \left\langle m, e_{i_{1}}-e_{i_{0}}\right\rangle>0 \\
\left(s_{1} t_{0}\right)^{1+\left\langle m, e_{i_{k+1}}\right\rangle}\left(s_{1} \cdots s_{k+1}\right)^{\left\langle m, e_{i_{0}}-e_{i_{1}}\right\rangle} & \text { if } & \left\langle m, e_{i_{1}}-e_{i_{0}}\right\rangle<0 .
\end{array}\right.
$$

When a $V$-manifold (orbifold) is embedded into another $V$-manifold it need not to be a $V$-submanifold (see Remark 3.3 in $[\mathrm{BC}]$ ). Magically, again, the complete intersection which represents the $V$-manifold $X$ in the toric variety $\mathbf{P}_{\Sigma\left(\Gamma^{*}\right)}$ is quasismooth, i.e., $V$-submanifold, if $X$ were generic enough (at least nondegenerate).

Proposition 4.4. If $X$ is a semiample anticanonical nondegenerate hypersurface defined by a homogeneous polynomial

$$
f(x)=\sum_{m \in \Delta \cap M} a_{m} \prod_{i=1}^{n} x_{i}^{1+\left\langle m, e_{i}\right\rangle}
$$

in a complete simplicial toric variety $\mathbf{P}_{\Sigma}$ as in Theorem 4.2, then the complete intersection variety $X$ described in Remark 4.3 is quasismooth in the toric variety $\mathbf{P}_{\Sigma\left(\Gamma^{*}\right)}$

Proof. To show that the complete intersection is quasismooth we need to check if the Jacobian matrix consisting of partial derivatives of defining polynomials has maximal possible rank on the complete intersection. In our case it will essentially suffice to look at the part of this matrix corresponding to the derivatives with respect to $s_{1}, \ldots, s_{k+1}, t_{0}, \ldots, t_{k}$ :

$$
\left(\begin{array}{cccccccccccc}
t_{0} & -t_{1} & 0 & 0 & \cdots & 0 & s_{1} & -s_{2} & 0 & 0 & \cdots & 0 \\
0 & t_{1} & -t_{2} & 0 & \cdots & 0 & 0 & s_{2} & -s_{3} & 0 & \cdots & 0 \\
0 & 0 & t_{2} & -t_{3} & \cdots & 0 & 0 & 0 & s_{3} & -s_{4} & \cdots & 0 \\
\vdots & & & \ddots & \ddots & \vdots & \vdots & & & \ddots & \ddots & \vdots \\
0 & 0 & 0 & \cdots & t_{k-1} & -t_{k} & 0 & 0 & 0 & \cdots & s_{k} & -s_{k+1} \\
\frac{\partial \tilde{f}}{\partial s_{1}} & & & \cdots & & \frac{\partial \tilde{f}}{\partial s_{k+1}} & \frac{\partial \tilde{f}}{\partial t_{0}} & & & \cdots & & \frac{\partial \tilde{f}}{\partial t_{k}}
\end{array}\right)
$$

where $\tilde{f}$ denotes the polynomial on the right hand side of the equation (8). What is missing in the above matrix compared to the whole Jacobian matrix are the partial derivatives with respect to $y_{i}$ for $e_{i} \notin \Gamma^{*}$.

First, note that $\partial \tilde{f} / \partial y_{i}$ coincides with $\partial f / \partial x_{i}$ for $e_{i} \notin \Gamma^{*}$ after transformation (4). So, if one of such $\partial f / \partial x_{i}$ does not vanish then the Jacobian matrix will have the maximal rank equal to $k+1$, since the above matrix without the last row is 
already shown to have the maximal rank on the intersection in the proof of Theorem 4.2. Now, since $X$ is nondegenerate, polynomials $x_{1} \partial f / \partial x_{1}, \ldots, x_{n} \partial f / \partial x_{n}$ do not vanish simultaneously on $\mathbf{P}_{\Sigma}$. Hence, we can assume that one of the following polynomials $x_{i_{0}} \partial f / \partial x_{i_{0}}, \ldots, x_{i_{k+1}} \partial f / \partial x_{i_{k+1}}$ does not vanish on $\mathbf{P}_{\Sigma}$. Then, since every $x_{i_{l}} \partial f / \partial x_{i_{l}}$, for $l=1, \ldots, k$, can be expressed via $x_{i_{0}} \partial f / \partial x_{i_{0}}$ and $x_{i_{k+1}} \partial f / \partial x_{i_{k+1}}$ by Euler formulas (see Definition 3.9 in [BC]), the polynomial $x_{i_{0}} \partial f / \partial x_{i_{0}}$ or $x_{i_{k+1}} \partial f / \partial x_{i_{k+1}}$ is not zero on $X$.

Second, note that $x_{i_{0}} \partial f / \partial x_{i_{0}}$ coincides with the polynomial

$$
s_{1} \frac{\partial \tilde{f}}{\partial s_{1}}+\cdots+s_{k+1} \frac{\partial \tilde{f}}{\partial s_{k+1}}
$$

after the substitution (4). (This is rather a straightforward calculation that we leave to the reader.) Similarly, $x_{i_{k+1}} \partial f / \partial x_{i_{k+1}}$ corresponds to

$$
t_{0} \frac{\partial \tilde{f}}{\partial t_{0}}+\cdots+t_{k} \frac{\partial \tilde{f}}{\partial t_{k}} .
$$

Now, as in the proof of the above theorem, a point on the complete intersection in $\mathbf{P}_{\Sigma\left(\Gamma^{*}\right)}$ will either have all coordinates $s$ and $t$ not equal to zero, or we can assume that there is $l$ between 0 and $k+1$ such that $t_{j} \neq 0$, for $j \geq l, t_{0}=\cdots=t_{l-1}=0$, $s_{j} \neq 0$, for $j \leq l-1$, and $s_{l+1}=\cdots s_{k+1}=0$. In the latter case, it will suffice to have that one of the partial derivatives

$$
\frac{\partial \tilde{f}}{\partial s_{1}}, \ldots, \frac{\partial \tilde{f}}{\partial s_{l}}, \frac{\partial \tilde{f}}{\partial t_{l}}, \ldots \frac{\partial \tilde{f}}{\partial t_{k}}
$$

in the last row of the matrix does not vanish. But if they do vanish, then both $x_{i_{0}} \partial f / \partial x_{i_{0}}$ and $x_{i_{k+1}} \partial f / \partial x_{i_{k+1}}$ will be zero by the above representation in terms of the sums, which would give a contradiction with nondegeneracy of $X$. If all $s$ and $t$ do not vanish, then multiply each of column of the above matrix, corresponding to the partial derivative with respect to a variable $s$ or $t$, by that variable. This procedure will not change the rank of the matrix. The left half of the new matrix will be

$$
\left(\begin{array}{cccccc}
s_{1} t_{0} & -s_{2} t_{1} & 0 & 0 & \cdots & 0 \\
0 & s_{2} t_{1} & -s_{3} t_{2} & 0 & \cdots & 0 \\
0 & 0 & s_{3} t_{2} & -s_{4} t_{3} & \cdots & 0 \\
\vdots & & & \ddots & \ddots & \vdots \\
0 & 0 & 0 & \cdots & s_{k} t_{k-1} & -s_{k+1} t_{k} \\
s_{1} \frac{\partial \tilde{f}}{\partial s_{1}} & & & \cdots & & s_{k+1} \frac{\partial \tilde{f}}{\partial s_{k+1}}
\end{array}\right)
$$

Since $s_{j+1} t_{j}=s_{j} t_{j-1}$, for $j=1, \ldots, k$, adding in succession to the second column the first one, then to the third the second one, and so on, will produce a new matrix:

$$
\left(\begin{array}{cccccc}
s_{1} t_{0} & 0 & 0 & \cdots & & 0 \\
0 & s_{1} t_{0} & 0 & \cdots & & 0 \\
0 & 0 & s_{1} t_{0} & \cdots & & 0 \\
\vdots & & & \ddots & & \vdots \\
0 & & \cdots & & s_{1} t_{0} & 0 \\
& & \cdots & & & *
\end{array}\right)
$$


where $s_{1} t_{0} \neq 0$ on the diagonal and $*$ is the polynomial $s_{1} \frac{\partial \tilde{f}}{\partial s_{1}}+\cdots+s_{k+1} \frac{\partial \tilde{f}}{\partial s_{k+1}}$, which by symmetry we can assume doesn't vanish. Hence, the original Jacobian matrix has the maximal rank.

The second main result shows that deformations of the toric variety $\mathbf{P}_{\Sigma}$ and its Calabi-Yau hypersurface are also realized as complete intersections inside another toric variety. The proof of the theorem also shows a relation of these deformations to the deformations constructed in [M5].

Theorem 4.5. Let $X$ be a semiample anticanonical hypersurface, defined by a homogeneous polynomial

$$
f(x)=\sum_{m \in \Delta \cap M} a_{m} \prod_{i=1}^{n} x_{i}^{1+\left\langle m, e_{i}\right\rangle}
$$

in $S(\Sigma)$, in a complete simplicial toric variety $\mathbf{P}_{\Sigma}$ as in Theorem 4.2. Then the following family of complete intersections in $\mathbf{P}_{\Sigma\left(\Gamma^{*}\right)}$ given by

$$
s_{j+1} t_{j}=s_{j} t_{j-1}+\sum_{u \in \operatorname{int}(\Gamma)} \lambda_{u, j} \prod_{e_{i} \notin \Gamma^{*}} y_{i}^{\left\langle u, e_{i}\right\rangle}, \quad \text { for } j=1, \ldots, k,
$$

and equation (8) are non-polynomial deformations of $X$, corresponding to

$$
\bigoplus_{e_{l} \in \operatorname{int}\left(\Gamma^{*}\right)}\left(S /\left\langle x_{l}\right\rangle\right)_{\beta_{1}^{\sigma}} \subset H^{1}\left(X, \mathcal{T}_{X}\right)
$$

from Proposition 2.1 by the Kodaira-Spencer map. Polynomial deformations of $X$ are parametrized by the coefficients $a_{m}$ in (8).

Proof. Using a different method in [M5], we already constructed 1-parameter nonpolynomial deformations of the Calabi-Yau hypersurface $X$, which are induced by the deformations of the toric variety $\mathbf{P}_{\Sigma}$. By the Kodaira-Spencer map, the corresponding infinitesimal deformations in $H^{1}\left(X, \mathcal{T}_{X}\right)$ have also been found in Section 3 of that paper. The essential work left is to show that those deformations of the toric variety coincide with the following 1-parameter subfamilies of deformations of $\mathbf{P}_{\Sigma}$, which are complete intersections in $\mathbf{P}_{\Sigma\left(\Gamma^{*}\right)}$ :

$$
s_{j+1} t_{j}=s_{j} t_{j-1}, \text { for } j \neq l \text { and } s_{l+1} t_{l}=s_{l} t_{l-1}+\lambda \prod_{e_{i} \notin \Gamma^{*}} y_{i}^{\left\langle u, e_{i}\right\rangle},
$$

where $u \in \operatorname{int}(\Gamma)$. After this, we only need to check that everything carries over for the hypersurface.

Recall the construction from [M5]. Let $U_{0}^{l}=\left\{x \in \mathbf{P}_{\Sigma}: \prod_{k>j} x_{l_{k}} \neq 0\right\}$ and $U_{1}^{l}=\left\{x \in \mathbf{P}_{\Sigma}: \prod_{k<j} x_{l_{k}} \neq 0\right\}$ be the open cover of the toric variety $\mathbf{P}_{\Sigma}$. The deformation of $\mathbf{P}_{\Sigma}$ is defined by regluing $U_{0}^{l}$ and $U_{1}^{l}$ along their intersection $U_{0}^{l} \cap U_{1}^{l}$. More precisely, the 1-parameter family $\mathcal{P}$ is obtained by gluing $U_{0}^{l} \times \mathbb{A}^{1}$ and $U_{1}^{l} \times \mathbb{A}^{1}$ along $U_{0}^{l} \cap U_{1}^{l} \times \mathbb{A}^{1}$ by the identification:

$$
\left(x_{1}, \ldots, x_{l}, \ldots, x_{n}, \lambda\right) \mapsto\left(x_{1}, \ldots, x_{l}-\lambda x_{l} \prod_{i=1}^{n} x_{i}^{\left\langle u, e_{i}\right\rangle}, \ldots, x_{n}, \lambda\right) .
$$

(Note: instead of $2 \lambda$ in [M5] we have $\lambda$ here for simplification.)

The complete intersections (9) can also be put into a family over $\mathbb{A}^{1}$ :

$$
\mathcal{P}^{\prime}=\left\{(y, \lambda) \in \mathbf{P}_{\Sigma\left(\Gamma^{*}\right)} \times \mathbb{A}^{1}:(9) \text { holds for }(y, \lambda)\right\} .
$$


Let us show that the families $\mathcal{P}$ and $\mathcal{P}^{\prime}$ are isomorphic. The second family $\mathcal{P}^{\prime}$ admits a covering by two open subsets: $\tilde{U}_{0}^{l}$ and $\tilde{U}_{1}^{l}$ defined by $t_{l} \neq 0$ and $s_{l} \neq 0$, respectively. Consider the following isomorphism $U_{0}^{l} \times \mathbb{A}^{1} \cong \tilde{U}_{0}^{l}$, defined by composing $\phi_{\Gamma^{*}} \times$ id with the map on $\tilde{U}_{0}^{l}$ which changes only the variables

$$
s_{j} \mapsto s_{j}-\frac{\lambda}{t_{j-1}} \prod_{e_{i} \notin \Gamma^{*}} y_{i}^{\left\langle u, e_{i}\right\rangle}
$$

for $j \geq l+1$. Note that these $t_{j-1} \neq 0$ since $t_{l} \neq 0$, as in the proof of Theorem 4.2. Similarly, $U_{1}^{l} \times \mathbb{A}^{1} \cong \tilde{U}_{1}^{l}$, which is a composition of $\phi_{\Gamma^{*}} \times$ id with the map on $\tilde{U}_{1}^{l}$ which changes

$$
t_{j} \mapsto t_{j}+\frac{\lambda}{s_{j+1}} \prod_{e_{i} \notin \Gamma^{*}} y_{i}^{\left\langle u, e_{i}\right\rangle}
$$

for $j \leq l-1$. Since $u \in \operatorname{int}(\Gamma)$, by the properties of the dual faces $\left\langle u, e_{i}\right\rangle=-1$ for $e_{i} \in \Gamma^{*}$. It is straightforward to check that the isomorphim $U_{0}^{l} \times \mathbb{A}^{1} \cong \tilde{U}_{0}^{l}$ is the composition of (10) with the above defined $U_{1}^{l} \times \mathbb{A}^{1} \cong \tilde{U}_{1}^{l}$.

The 1-parameter family $\mathcal{X} \subset \mathcal{P}$ corresponding to the deformation of the CalabiYau hypersurface $X$, determined by

$$
f(x)=\sum_{m \in \Delta \cap M} a_{m} \prod_{i=1}^{n} x_{i}^{1+\left\langle m, e_{i}\right\rangle},
$$

is given by the hypersurfaces in $U_{\varepsilon}^{l} \times \mathbb{A}^{1}, \varepsilon=0,1$, corresponding to

$$
f_{\lambda}^{\varepsilon}(x)=\sum_{m \in \Delta} a_{m} \prod_{i=1}^{n} x_{i}^{-1+\left\langle m, e_{i}\right\rangle}\left(1+\left((-1)^{\varepsilon}+c_{m}\right) \frac{\lambda}{2} \prod_{i=1}^{n} x_{i}^{\left\langle u, e_{i}\right\rangle}\right)^{-1+\left\langle m, e_{l}\right\rangle},
$$

where

$$
c_{m}=\left\{\begin{array}{clll}
1 & \text { if } & \left.\left\langle m, e_{l_{0}}-e_{l}\right\rangle\right)>0 \\
0 & \text { if } & \left.\left\langle m, e_{l_{0}}-e_{l}\right\rangle\right)=0 \\
-1 & \text { if } & \left.\left\langle m, e_{l_{0}}-e_{l}\right\rangle\right)<0
\end{array}\right.
$$

One can check that these hypersurfaces are compatible with respect to the identification (10). Moreover, polynomials $f_{\lambda}^{\varepsilon}(x)$ will both transfer to the polynomial

$$
\sum_{m \in \Delta \cap M} a_{m} X_{m} \prod_{e_{i} \notin \Gamma^{*}} y_{i}^{1+\left\langle m, e_{i}\right\rangle}
$$

in (8) by the already defined isomorphisms $U_{\varepsilon}^{l} \times \mathbb{A}^{1} \cong \tilde{U}_{\varepsilon}^{l}$. Hence, this deformation of $X$ is isomorphic to the intersection of (9) with (8).

The fact that the polynomial deformations of $X$ correspond to deformations of $f$ is shown in [M5, Section 1].

Remark 4.6. In the above theorem, the non-polynomial deformations for a minimal Calabi-Yau hypersurface $X$ correspond to the term $l^{*}(\Gamma) l^{*}\left(\Gamma^{*}\right)$ in Proposition 2.2 .

Remark 4.7. In the Batyrev-Borisov mirror symmetry construction (see [BBo]), the Calabi-Yau complete intersections are nef. In our construction, we can assume that the ambient toric variety $\mathbf{P}_{\Sigma\left(\Gamma^{*}\right)}$ is complete (by compactifying it if necessary). However, in most cases, the complete intersection in Theorem 4.5 will not be nef. Here is the simplest example of such a Calabi-Yau complete intersection. Let $\Sigma$ 
be the fan with cones formed by a triangulation of the reflexive polytope whose vertices are

$$
\{(-2,-2,-3,-2),(1,0,0,0),(0,1,0,0),(0,0,1,0),(0,0,1,2)\} \text {. }
$$

Except for the origin there are three more lattice points $(0,0,1,1),(-1,-1,-1,-1)$ and $(-1,-1,-1,0)$ in this polytope that lie in the middle of the edges of the twodimensional face with vertices at $(0,0,1,0),(0,0,1,2)$ and $(-2,-2,-3,-2)$. We can apply our construction to the edge between $e_{i_{0}}=(0,0,1,0)$ and $e_{i_{2}}=(0,0,1,2)$ with $e_{i_{1}}=(0,0,1,1)$ as the only interior lattice point. By the formulas (5), we get a new fan $\Sigma\left(\Gamma^{*}\right)$ with the set of minimal integral generators at the points

$$
\begin{aligned}
& (-2,-2,-3,-2,0),(1,0,0,0,0),(0,1,0,0,0),(-1,-1,-1,-1,0), \\
& (-1,-1,-1,0,0), v=(0,0,0,0,1), e_{i_{0}}-v=(0,0,1,0,-1), \\
& v+\frac{e_{i_{2}}-e_{i_{0}}}{2}=(0,0,0,1,1), e_{i_{0}}-v+\frac{e_{i_{2}}-e_{i_{0}}}{2}=(0,0,1,1,-1) .
\end{aligned}
$$

However, the convex hull of these points in $\mathbb{Z}^{5}$ is not a reflexive polytope. On the other hand, if the hypersurfaces defining the complete intersection in $\mathbf{P}_{\Sigma\left(\Gamma^{*}\right)}$ were nef, then the sum of the degrees of these hypersurfaces was also nef and anticanonical, by the construction. But in this case, by the uniqueness result of [M2, Proposition 1.2] and [B, Theorem 4.1.9], the variety $\mathbf{P}_{\Sigma\left(\Gamma^{*}\right)}$ must be a partial crepant resolution of a Fano toric variety, in which case, the convex hull of the generators of $\Sigma\left(\Gamma^{*}\right)$ form a reflexive polytope. Hence, we get a contradiction with the assumption that the complete intersection was nef. This example is very important. It indicates that we have to study non-nef Calabi-Yau complete intersections in toric varieties.

Acknowledgement. The author would like to thank the Max-Planck Institute for Mathematics in Bonn for an opportunity to finish this paper.

\section{REFERENCES}

[B] V. V. Batyrev, Dual polyhedra and mirror symmetry for Calabi-Yau hypersurfaces in toric varieties, J. Algebraic Geometry 6 (1994), 493-535.

[BBo] V. V. Batyrev and L. A. Borisov, "Dual Cones and Mirror Symmetry for Generalized Calabi-Yau Manifolds" in Mirror Symmetry II, (B. Green and S.-T. Yau, eds.), AMS/IP Stud. Adv. Math. 1, AMS, Providence, RI, 1997, 71-86.

[BC] V. V. Batyrev and D. A. Cox, On the Hodge structure of projective hypersurfaces in toric varieties, Duke Math. J. 75 (1994), 293-338.

[C] D. Cox, The homogeneous coordinate ring of a toric variety, J. Algebraic Geom. 4 (1995), 17-50.

[CK] D. Cox and S. Katz, Algebraic Geometry and Mirror Symmetry, Math. Surveys Monogr. 68, Amer. Math. Soc., Providence, 1999.

[CadFKMo] Mirror symmetry for two-parameter models - I, P. Candelas, X. de la Ossa, A. Font, S. Katz and D.R. Morrison, Nucl. Phys. B416 (1994), 481.

[D] V. Danilov, The geometry of toric varieties, Russian Math. Surveys 33 (1978), 97154 .

[F] W. Fulton, Introduction to toric varieties, Princeton Univ. Press, Princeton, NJ, 1993.

[KS] M. Kreuzer, H. Skarke, PALP: A Package for Analyzing Lattice Polytopes with Applications to Toric Geometry, preprint/math.NA/0204356.

[M1] A. R. Mavlyutov, Cohomology of complete intersections in toric varieties, Pacific J. Math. 191 (1999), 133-144. 
[M2] S Semiample hypersurfaces in toric varieties, Duke Math. J. 101 (2000), 85116.

[M3] _ The Hodge structure of semiample hypersurfaces and a generalization of the monomial-divisor mirror map, in Advances in Algebraic Geometry Motivated by Physics (ed. E. Previato), Contemporary Mathematics, 276, 199-227.

[M4] , "On the chiral ring of Calabi-Yau hypersurfaces in toric varieties", Comp. Math., to appear, preprint /math.AG/0010318.

[M5] Deformations of Calabi-Yau hypersurfaces arising from deformations of toric varieties, preprint math.AG/0309239.

[Od] T. Oda, Convex Bodies and Algebraic geometry, Springer-Verlag, Berlin, 1988.

Department of Mathematics, Indiana University, Bloomington, IN 47405, USA.

E-mail address: amavlyut@indiana.edu 